\title{
IX. West- oder Ostdeutschland?
}

Noch Anfang der 50er Jahre hatten einzelne Vertreter der regierenden sozialistischen Arbeiterpartei Israels (Mapai) die deutschen Sozialisten den bürgerlichen Politikern Westdeutschlands vorgezogen. Die Politiker der CDU/CSU und FDP waren den israelischen Politikern ihrer potentiell belasteten Vergangenheit und ihrem umstrittenen Verhalten nach 1945 wegen suspekt. Sofern zwischen Deutschen überhaupt differenziert wurde, fühlten sich die israelischen Sozialisten den deutschen Sozialisten in beiden deutschen Staaten am nächsten. Die Vorbehalte gegenüber Deutschland waren zwar, wie wir gesehen haben, nicht verschwunden, doch immerhin betrachteten die israelischen Sozialisten die jüdischen Sozialisten und Kommunisten, die sich dazu entschlossen hatten, am Aufbau des „ersten deutschen sozialistischen Staates", also der DDR, teilzunehmen, ohne das Leid der Vergangenheit aus den Augen zu verlieren, als Brücke zwischen den Völkern. Tatsächlich waren die Juden im politischen Establishment und unter den Eliten des Ostens zahlreicher vertreten als im Westen. Der jüdische Bundestagsabgeordnete Altmaier, von Schumacher sorgfältig ausgewählt, und seine Abgeordnetenkollegin Jeanette Wolff bildeten im Westen eher die Ausnahme. Jüdische Rückkehrer, auch jene, die auf Einladung nach Westdeutschland zurückgekehrt waren, berichteten über die großen Schwierigkeiten der Wiedereingliederung. Den jüdischen Sozialisten in Palästina und später in Israel war nicht entgangen, daß die Vorkriegsparteigenossen und Antinazis im Osten willkommener waren als im Westen.

\section{Israelische Erwartungen gegenüber Pankow}

Als der Staat Israel gegründet wurde, waren die israelisch-sowjetischen Beziehungen relativ gut, und der Ostblock griff dem jungen Staat in seinem Überlebenskampf unter die Arme. Entsprechend groß war in Israel damals die Sympathie für die Sowjetunion. Die Sowjetische Besatzungszone gab sich Israel gegenüber freundlich und profitierte vom israelischen Wohlwollen für Moskau.

Bereits im Frühjahr 1948, noch vor der Gründung des Staates Israel, wurde Chaim Yachil vom Vorsitzenden der SED, Otto Grotewohl, zum Gespräch empfangen, und zusammen mit Eliahu Livneh traf er später auch die DDR-Funktionäre Leo Zuckermann und Hilde Benjamin. Zuckermann, damals persönlicher Sekretär des zweiten SED-Vorsitzenden Wilhelm Pieck und später, während der Säuberungen in den fünfziger Jahren, aus der DDR geflüchtet, hatte am 17. April 1948 einen Artikel in der westdeutschen Weltbübne veröffentlicht, in dem er für das Recht der Juden auf kollektive und individuelle Entschädigung eintrat und für die internationale Anerkennung des zukünftigen jüdischen Staates votierte. ${ }^{1} \mathrm{Die}$

1 ZuckermanN, Restitution und Wiedergutmachung. Bislang konnte in den Archiven der DDR kein Hinweis auf diese Gespräche gefunden werden. 
Bevölkerung der Sowjetischen Besatzungszone (SBZ) brachte dem ums Überleben kämpfenden Jischuv während des Unabhängigkeitskrieges beträchtliche Sympathien entgegen. ${ }^{2}$ Der kommunistische Funktionär Paul Merker äußerte sich in einem Zeitungsartikel mit dem Titel „Der neue Staat des jüdischen Volkes“, der am 24. Februar $1948 \mathrm{im}$ Neuen Deutschland erschien, positiv zum noch nicht gegründeten Staat Israel und sagte Hilfe der "deutschen demokratischen Kräfte“ $\mathrm{zu}^{3}$

In scheinbarem Gegensatz zur westdeutschen SPD, die dem neugegründeten Israel mit einer gewissen Kühle begegnete, demonstrierte die ostdeutsche Führung eine positive, wenn auch vorsichtig abgewogene Haltung gegenüber dem jüdischen Staat. ${ }^{4}$ Über das Paria-Dasein der DDR innerhalb des Ostblocks wurde bislang nur wenig geschrieben. In den ersten Jahren ihres Bestehens versuchte die DDR verzweifelt aus ihrer fast völligen internationalen Isolation auszubrechen. Noch in den frühen fünfziger Jahren bemühte sich die DDR krampfhaft um die Verbesserung ihrer Beziehungen zu Israel, selbst mit ausgefallenen Mitteln. Ludwig Lewy, ein nach Israel ausgewanderter jüdischer Journalist ostdeutscher Herkunft wurde vom DDR-Außenministerium mit der Übermittlung von Botschaften an die israelische Regierung beauftragt. Diese Noten enthielten äußerst großzügige Angebote ohne nennenswerte Gegenforderungen: Individuelle Entschädigung, nicht an Devisen gebundenen Handel und die offizielle Anerkennung eines israelischen Konsulats in Berlin, an einem Standort nach freier Wahl. ${ }^{5}$ Die kurze Zeit später versandte Note eines Vertreters der Jewish Agency for Palestine (JAFP) bereitete Lewys Bemühungen dann ein abruptes Ende: „Es werden keine Verhandlungen über jüdische Forderungen gegenüber Ostdeutschland geführt, und es besteht auch keine Hoffnung auf solche Verhandlungen. Dieses Angebot weiter zu verfolgen, ist deshalb zwecklos. “6

Israels allmähliche Annäherung an den Westen und die zunehmende Feindseligkeit der Sowjetunion gegenüber dem jüdischen Staat und den Juden machte eine Verständigung zwischen der DDR und Israel immer schwieriger. Trotzdem wirft die kühle israelische Reaktion zum Versuchsballon der DDR einige Fragen auf. Einen Anhaltspunkt zu ihrer Klärung bietet die Korrespondenz des American Jewish Committee (AJC) in den Jahren, als die israelische Deutschfeindlichkeit noch sprichwörtlich war. Man stößt dabei auf Sätze wie den folgenden: "Auch in diesem Fall scheinen sich die westlichen und amerikanischen Interessen (einschließlich unserer Interessen) den Beziehungen zwischen Israel und dem ÖSTLICHEN [Hervorhebung im Original] Deutschland viel stärker zu widersetzen als zwischen Israel und dem Westen. " gegenüber eine feindselige Haltung einzunehmen begann, stand der jüdische Staat

2 EsCHWEGE, Die jüdische Bevölkerung, S. 90.

3 Vgl. die ähnliche Erklärung bei WINROw, East Germany, S. 32.

4 SHAFIR, Ha yad ha-musheteth, S. 41-44.

5 Note von Ludwig Lewy, ostdeutsches Außenministerium, CZA, S 35/198. Lewy kontaktierte Georg Landauer: Al Hamishmar (Tel Aviv) vom 30. 3. 1951.

$6 \mathrm{H}$. Gerling an Ludwig Lewy vom 8. 1. 1951, CZA, S35/198.

7 Hevesi an Segal vom 21. 12. 1949, YIVO, AJC, FAD-1, Box 36. 
bereits vor dem Dilemma, sich zwischen dem kapitalistischen Westdeutschland und dem kommunistischen Ostdeutschland entscheiden zu müssen. In der zweigeteilten Welt, zwischen zwei Blöcken und zwei Ideologien, hatten Israels Neutralitätsbestrebungen langfristig keine Chance.

Vitale wirtschaftliche und politische Interessen, militärische Verbindungen, die Israel zugeneigte jüdische Diaspora sowie traditionelle politische und ideologische Neigungen gaben den Ausschlag für den Westen. Das hatte die allmähliche Abkehr von der Blockfreiheit und die Annäherung an die Vereinigten Staaten und ihre Verbündeten fast zwangsläufig zur Folge. Da sich die Bundesrepublik zu einem der wichtigsten Verbündeten der USA entwickelte, blieb der israelischen Regierung faktisch gar keine andere Wahl, wie der Rechtsberater des israelischen Außenministeriums Shabtai Rosenne in einem Gutachten über den „Kriegszustand mit Deutschland " bemerkte. Rosenne war darüber im klaren, daß es sich um eine politische Entscheidung [die Wahl zwischen den deutschen Staaten] mit "schwerwiegendsten politischen Konsequenzen" handle. ${ }^{8}$ Um diesen auszuweichen, schlug Rosenne in seiner hebräischen Zusammenfassung des Gutachtens direkte Verhandlungen etwa durch eine in Ostberlin akkreditierte offizielle Vertretung vor. ${ }^{9}$ Welch ausgefallene Idee! Weshalb sollte die israelische Regierung, die zögerte, direkte Gespräche mit Bonn zu führen, dies ausgerechnet mit Pankow tun? Die israelischen Diplomaten hatten jedenfalls Anweisung, nicht mit ihren ostdeutschen Kollegen zu kommunizieren. ${ }^{10}$

Gleichwohl richtete Israel Appelle an die Sowjetunion, die von ihr abhängige DDR zu Gesprächen zu bewegen. Diese zeitigten ähnliche Resultate wie die entsprechenden Vorstöße im Westen. Die Sowjetunion berief sich auf die Souveränität der DDR und verwies die israelischen Regierung direkt an Ostberlin. Die Motive der UdSSR und der USA glichen sich stark: Beide Großmächte waren daran interessiert, ihren Schützlingen internationale Anerkennung zu verschaffen oder, mit anderen Worten, ihnen einen "Platz in der Völkerfamilie" $\mathrm{zu}$ sichern.

Am 13. März 1951 sandte Israel eine Note an die Sowjetunion, deren Inhalt sich kaum vom Inhalt der israelischen Noten an die westlichen Alliierten unterschied. Der Kreml antwortete nicht. Inoffiziell zeigte er der israelischen Regierung die kalte Schulter und forderte sie mit dem Hinweis auf die Souveränität der DDR einmal mehr auf, sich direkt an Ostberlin zu wenden. Zudem wies die Sowjetunion entsprechend den Argumenten der westlichen Großmächte auf die fehlende rechtliche Grundlage für die israelischen Ansprüche hin. In Yalta und Potsdam hätten nur die Alliierten Reparationen zugesprochen erhalten, und Israel sei in diesen Dokumenten nicht erwähnt, lautete die sowjetische Antwort. Der östliche Teil Deutschlands könne somit für nichtexistierende Ansprüche auch keine Ver-

8 Ausarbeitung des Rechtsberaters Shabtai Rosenne "Ansicht über die Note vom 24. 10. 1950 der drei Mächte betr. die Beendigung des Kriegszustands mit Deutschland“ vom November 1950, ISA, 344/15.

9 Der Rechtsberater des Außenministeriums an das Sekretariat des Ministers für den Minister und den Generaldirektor vom 30.11. 1950, ISA, 344/15.

10 Der Leiter der Abteilung Osteuropa an die Konsularabteilung vom 3. 4. 1950, ISA, 2539/ $6 a$. 
pflichtungen übernehmen. Nur ein Friedensvertrag könne dieses Problem lösen. ${ }^{11}$ Als die westlichen Großmächte von den israelischen Anstrengungen erfuhren, Reparationen von der DDR zu erlangen, äußerten sie die Befürchtung, die Sowjetunion könnte die Gelegenheit nutzen, um die Reparationsthematik neu aufzurollen. Tatsächlich versuchte der Kreml Israels Ansprüche als Vorwand für die Einberufung einer Friedenskonferenz, selbstverständlich gemäß seinen Bedingungen, zu nutzen. ${ }^{12}$

Mehrere israelische Vertreter rieten zum direkten Gespräch mit der ostdeutschen Regierung. Doch Pankows Weigerung, eine Erklärung im Sinne der Adenauers vom 27. September 1951 abzugeben, scheint die israelische Regierung daran gehindert zu haben. Zudem zeigte die ostdeutsche Regierung keinerlei Bereitschaft, die sich in der DDR oder im Ausland aufhaltenden NS-Opfer individuell zu entschädigen, womit ein weiterer Grund für die Bevorzugung der Bundesrepublik durch Israel gegeben war. Das DDR-Regime zögerte nicht, auch jüdisches Eigentum zu beschlagnahmen und zu verstaatlichen und dies mit der kommunistischen Doktrin zu rechtfertigen. Da die jüdischen Bürger zweimal unter dieser drakonischen Maßnahme zu leiden hatten, einmal unter dem Nationalsozialismus und ein weiteres Mal unter den Kommunisten, war das Faktum, daß diese Politik alle Bürger traf, ein schwacher Trost. Die erneute Enteignung und die Weigerung, geraubtes Eigentum zurückzuerstatten, war ein weiterer harter Schlag für die stark dezimierte Gemeinde.

Die DDR begründete ihre Weigerung, Entschädigung zu entrichten, zunächst mit fehlenden Mitteln. ${ }^{13}$ Von den Zerstörungen des Zweiten Weltkriegs stärker heimgesucht als Westdeutschland, von der Sowjetunion mit umfangreichen Demontageforderungen konfrontiert und zur Aufnahme Hunderttausender sogenannter Volksdeutscher gezwungen, war die DDR kaum in der Lage, Mittel für Schilumim oder andere Arten von Entschädigung bereitzustellen. Später wurden die Schilumim dann als „kapitalistische Verschwörung" gegen den sozialistischen Staat gebrandmarkt, die darauf abziele, den wirtschaftlichen Wiederaufbau zu verhindern oder, noch schlimmer, als amerikanisch-israelischer Komplott gegen die arabischen Völker mit deutschem Geld. ${ }^{14}$ Wie auch immer, der dringend auf Wirtschaftshilfe angewiesene Staat Israel konnte sich keine Hoffnung auf Unterstützung aus der DDR machen, der schlicht das Geld dazu fehlte.

Angesichts der Bonner Unterstützung kam Israel nicht umhin, die Maßnahmen zur internationalen Ächtung Ostdeutschlands durch die Bundesrepublik zu unterstützen. Jedenfalls wurde das in Bonn vom jüdischen Staat erwartet. Für die Bundesrepublik machten sich die Schilumim auch im Hinblick auf die Ostpolitik, nicht zuletzt dank des Verhaltens der DDR, bezahlt. Angesichts der Diskriminierung der NS-Opfer im Osten war die Schilumim ein Pfund, mit dem Bonn wu-

11 Telegramm Levavis an Shinnar vom 26. 3. 1952, ISA, 2511/17a; Ausarbeitung von Eliashiv, Moskau, "Über die Gespräche mit dem Sowjetdiplomaten Pushkin“ vom 18. 9. 1952, ISA, 2418/13.

12 Eliashiv an Sharett vom 10. 9. 1952, ISA, 2418/13; 2545/6.

13 Bergmann an Finanzminister Kaplan vom 3. 9. 1950, ISA, 2416/15a.

14 TIMM, Hammer, S. 131-133. 
chern konnte. Während im Westen auch nichtjüdische NS-Opfer entschädigt wurden, gingen die Opfer im Osten - bis zum Fall der Mauer oder bis zur Übersiedlung in den Westen - leer aus.

Doch aufmerksame Beobachter erkannten auch die Risiken: Einmal mehr erhielten die Juden eine „Sonderbehandlung“, wenn auch diesmal zu ihren Gunsten. Mit der bevorzugten Behandlung der jüdischen Opfer nahm man negative Reaktionen der Öffentlichkeit und anderer Opfergruppen in Kauf. Es überrascht deshalb nicht, daß Beobachter die "Arisierung “ der Wiedergutmachung forderten, d.h. nichtjüdischen Opfern gleiche Entschädigung zu zahlen. ${ }^{15}$ Gemeint waren natürlich vor allem die Hilfsbedürftigen im eigenen Land, die Kriegswitwen und -waisen, die Ausgebombten und die Vertriebenen.

Die Bevorzugung der jüdischen NS-Opfer war nicht ungefährlich, wie das Beispiel der DDR zeigt. Die Bonner Linie verstärkte die ostdeutsche Feindseligkeit gegenüber dem jüdischen Staat, dem Kollaboration mit den Neonazis vorgeworfen wurde. Wie bereits erwähnt, betrachtete der Ostblock Israel anfänglich als natürlichen Verbündeten im Kampf gegen Hitlers Erbe. Dies war eine Vorstellung, die sich aus osteuropäischer Perspektive immer mehr als Illusion erwies und schließlich ganz aufgegeben wurde. Als die Schilumimverhandlungen in die kritische Phase traten, erreichte die stalinistische „antikosmopolitische“ Kampagne ihren Höhepunkt. Jerusalem gab sich indes zurückhaltend in der Debatte über westdeutsche Tabuthemen, wie etwa die Oder-Neiße-Linie, die Saarfrage und sogar die Hallsteindoktrin. Eine dezidierte Antinazihaltung konnte sich die israelische Regierung nicht leisten und Bonns Politik gegen ehemalige Verbündete im Kampf gegen NS-Deutschland sowie andere NS-Opfer wollte sie nicht unterstützen. So versuchte die israelische Regierung diese Fragen so gut wie möglich zu meiden und sich dazu nicht öffentlich zu äußern. Denn jede Stellungsnahme hatte ihren Preis.

\section{Geheime Ränke}

Israel hatte bei den Verhandlungen in Wassenaar zwei Artikel vorgeschlagen, die die DDR direkt betrafen: eine Wiedervereinigungsklausel und eine Kontinuitätsklausel. Letztere wurde rasch fallengelassen. Der Deutschlandvertrag garantierte die Kontinuität bestehender Abkommen, beschränkte aber auch Bonns Handlungsfreiheit in Bezug auf neue Verpflichtungen. Aber auch die Wiedervereinigungsklausel, durch die Israel festzulegen versuchte, daß „bei einer Wiedervereinigung Deutschlands keine neuen Verhandlungen nötig seien, da Ostdeutschland ca. ein Drittel der Kapazität Gesamtdeutschlands habe und daher die ursprüngliche Forderung Israels bereits entsprechend herabgesetzt sei", stieß auf heftigen Widerstand von deutscher Seite. ${ }^{16}$ Die deutsche Delegation wurde angewiesen, in

15 Dr. Brückser an Max Bachmann vom 29. 12. 1951, PA, 244-13/II, 115202/51.

16 Niederschrift des Bundeswirtschaftsministeriums über die Sitzung des Rechts- und Redaktionsausschusses in Oud Wassenaar vom 25.6. 1952, BArch, B 102/7019. 
diesem Punkt hart zu bleiben und keine weiteren Forderungen im Zusammenhang mit der Wiedervereinigung zu akzeptieren, auch wenn dadurch das Abkommen scheitern sollte. Außerdem erhielt die deutsche Delegation den Auftrag, eine Verringerung des Anteils der Bundesrepublik an der gesamtdeutschen Verpflichtung von zwei Dritteln auf $60 \%$ zu erreichen. ${ }^{17}$

Die Haltung der Bundesregierung stützte sich auf drei Argumente: erstens, die Bundesrepublik erkennt keine Forderungen an, die das wiedervereinigte Deutschland betreffen. Solche Forderungen stehen im Widerspruch zum Deutschlandvertrag; zweitens, ein solcher Artikel stößt im Bundestag und in der Öffentlichkeit auf psychologische und politische Schranken und löst Kontroversen aus; drittens, die Wiedervereinigung wird eine teure Angleichung des Lebensniveaus in beiden Teilen Deutschlands erfordern. Die Bundesrepublik sollte deshalb weitere Verpflichtungen gegenüber dem Ausland vermeiden. ${ }^{18}$ Die deutsche Delegation blieb hart, und der Artikel wurde zusammen mit weiteren umstrittenen Artikeln gestrichen. Das bedeutet aber nicht, daß die Israelis ihren Schilumimanspruch gegenüber der DDR aufgaben. Er wurde nur zurückgestellt - und später wieder vorgebracht. Das Schilumimabkommen enthielt also keine Vorkehrungen für zukünftige Eventualitäten, mit Ausnahme von zwei Zusatzdokumenten, in denen sich der Staat Israel verpflichtete, auf weitere Ansprüche gegenüber der Bundesrepublik zu verzichten. Am 12. August $1952 \mathrm{kam}$ es im israelischen Außenministerium zur ersten internen Debatte über zukünftige Verhandlungen mit Ostdeutschland und zu ersten konkreten Vorbereitungen in dieser Angelegenheit. Man hoffte, Pankow zu Verhandlungen über Schilumim bewegen zu können. ${ }^{19}$

Da der Weg nach Pankow bekanntlich über Moskau führte, überreichte der israelische Gesandte in Moskau dem sowjetischen Vizeaußenminister Alexander S. Puschkin am 10. September 1952 eine Note, in der Israel Verhandlungen mit Ostdeutschland forderte. In einer ersten mündlichen Stellungnahme hieß Puschkin das israelische Begehren im Grundsatz gut, wies jedoch auf die Friedenskonferenz als geeignetes Forum für die Behandlung dieser Frage hin. ${ }^{20}$ Der sowjetische Vizeaußenminister versuchte Israel für die sowjetischen Standpunkte in den europäischen Konflikten zu gewinnen und skizzierte die sowjetische Haltung in der Schilumimfrage. Die DDR sei ein souveräner Staat und somit direkt zu kontaktieren, was wiederum mindestens einer faktischen Anerkennung gleichkäme. Ein Teil des geteilten Deutschland könne keine Verpflichtungen im Namen von ganz Deutschland übernehmen. Es sei deshalb im Interesse Israels, meinte Puschkin weiter, die Wiedervereinigungsbemühungen aktiv zu unterstützen. Jedes Separatabkommen mit Bonn bedeute, die Teilung zu legitimieren. Die deutschen Reparationen beruhten auf internationalen Abkommen, was von den israelischen

17 Protokoll über das Treffen von Shinnar, Keren, Abs und Wolf in London vom 19. 5. 1952, CZA, Z6/1022.

18 Niederschrift des Bundeswirtschaftsministeriums über die Sitzung des Rechts- und Redaktionsausschusses in Oud Wassenaar vom 25. 6. 1952, BArch, B 102/7019.

19 Der stv. Generaldirektor an den israelischen Minister in Moskau vom 12. 8. 1952, ISA, 2511/17a.

20 Shmuel Eliashiv an Sharett vom 10. 9. 1952, ISA, 2415/13. 
Ansprüchen nicht behauptet werden könne. In seiner Antwort an Eliashiv bezeichnete Puschkin die Londoner Schuldenkonferenz als weiteren Versuch, die Teilung Deutschlands zu verewigen. Der sowjetische Vertreter war offensichtlich ein Meister seines Handwerks. Er wußte über die kleinsten Einzelheiten Bescheid und charakterisierte den Anspruch der NS-Opfer auf Entschädigung als neuartige Forderung, worüber ein internationales Forum zu entscheiden habe. Ihr Suigeneris-Charakter erfordere die Übertragung der Frage an eine Friedenskonferenz. Mit anderen Worten, die Aussichten auf Entschädigung aus Ostdeutschland waren äußerst beschränkt.

Die israelische Regierung ließ sich von diesem Rückschlag aber nicht entmutigen und suchte neue Wege, um ihre Ansprüche durchzusetzen. Die Frage war, wie mit der ostdeutschen Regierung Gespräche geführt werden konnten, ohne den Eindruck der faktischen Anerkennung dieses Staates zu erwecken. Eine Möglichkeit war der Kontakt mit dem ersten Botschafter der DDR in Moskau, dem Sudetendeutschen Rudolf Appelt. Appelt hatte in der Vergangenheit jüdische Freunde gehabt, war mit der Frage bestens vertraut und unterstützte persönlich den jüdischen Anspruch auf Entschädigung. Israelische Diplomaten in Moskau betrachteten den DDR-Diplomaten deshalb als Hauptansprechpartner für zukünftige Vorstöße. Inzwischen sammelte Ostberlin jedoch zunächst einmal Munition für seine Attacken gegen Israel.

Ende September 1952 allerdings überraschte der DDR-Landwirtschaftsminister Ernst Goldenbaum die eigene Regierung und die politischen Beobachter mit der Feststellung, sein Land habe bislang keine Entschädigung an Juden entrichtet, da es von niemandem dazu aufgefordert worden sei. Man sei aber bereit, die Opfer des Nationalsozialismus zu entschädigen. ${ }^{21}$ Die Gründe für diese seltsame, aufrichtig anmutende Äußerung sind bis heute unklar. Hat der Minister die Politik seiner Regierung mißverstanden, war es ein Ablenkungsmanöver oder schlicht ein aufrichtiges Bekenntnis? Etwa ein Monat später, am 25. November 1952, meldete sich das SED-Organ Neues Deutschland nach längerem Schweigen zu diesem Thema mit einem Leitartikel unter dem Titel „SED lehnt Abkommen über Wiedergutmachung mit Israel ab“, worin das Abkommen als „Pakt zwischen deutschen und israelischen Kapitalisten" bezeichnet wurde. Die deutsche Industrie habe bereits vom Mord an Millionen Juden profitiert und wolle sich nun weiter bereichern, hieß es. Obwohl auch israelische Kapitalisten davon profitierten, so der Leitartikel im SED-Organ, gehe die Initiative ursprünglich von den USA aus, die ihre Stellung in der Region auf Kosten der Araber ausbauen wollten. Die Unabhängigkeit der arabischen Staaten stehe auf dem Spiel, und das Schilumimabkommen vergrößere das arabische Mißtrauen gegen Deutschland, folgerte das Neue Deutschland.

Das Sperrfeuer der Kritik gegen die Schilumim erreichte seinen Höhepunkt mit publikumswirksamen Appellen von SED-Generalsekretär Walter Ulbricht und von DDR-Ministerpräsident Otto Grotewohl an die Adresse der Bundesrepublik, die Schilumimzahlungen sofort einzustellen. Auf einer wenige Wochen nach

21 Dawar ('Tel Aviv) vom 22. 9. 1952; Notiz vom 23. 9. 1952, IJA, 222.0. 
dem Aufstand vom 17. Juni 1953 stattfindenden Massenkundgebung, an der auch der sowjetische Hochkommissar Wladimir Semjonov teilnahm, forderten die beiden DDR-Spitzenpolitiker die sofortige Einstellung sämtlicher westdeutscher Zahlungen, einschließlich der Schilumim, anderer Kompensationen und Vorkriegsschulden. Das wiedervereinte Deutschland werde keine finanziellen Forderungen aus dem Ausland annehmen, hieß es. ${ }^{22}$ Diese Aufforderung, die anschließend in einer offiziellen an die Bundesregierung gerichteten Note auch als Bedingung für die Wiedervereinigung genannt wurde, dürfte unter anderem bezweckt haben, die Bevölkerung von innenpolitischen Problemen abzulenken. ${ }^{23}$ Ostberlin kam in den folgenden Jahren mehrmals darauf zurück.

Das israelische Außenministerium verfolgte die Streiks in der DDR und den Aufstand vom 17. Juni aufmerksam und entsandte Yachil als Beobachter vor Ort. Sein Bericht enthält eine ausführliche Beschreibung der Geschehnisse und deren Hintergründe sowie einen zusammenfassenden Bericht zur Lage der Juden in der DDR. ${ }^{24}$ Eine Erklärung für das besondere Interesse der israelischen Führung an den Ereignissen in Ostdeutschland bieten die Quellen nicht. Es könnte etwa auf die besondere Sensibilität der sozialistischen Führung Israels für die dramatischen Vorgänge - die ersten dieser Art im Ostblock - in einem sozialistisch regierten Land zurückzuführen sein. Die Ereignisse in Ostdeutschland ließen indirekt auch Rückschlüsse über Westdeutschland zu und trugen zum neuen Deutschlandbild Ben Gurions bei. Möglicherweise sollte Yachils Bericht auch eine Grundlage für die Formulierung von Schilumimforderungen an die DDR bilden.

In den Jahren 1952 und 1953 gab es in den kommunistischen Staaten öffentliche Kampagnen gegen ideologische Dissidenten, Kommunisten, die in Ungnade gefallenen waren, und -Juden. Zu den Ausgestoßenen zählten unter anderem Rückkehrer, die sich während des Krieges im Westen aufgehalten hatten, eine Gruppe, die auch in der DDR zahlreich vertreten war. Einige dieser Rückkehrer waren Juden. Nachdem die israelische Regierung und führende jüdische Vertreter sich für den politischen und ideologischen Widersacher und „Klassenfeind“ in Bonn entschieden hatten, dürften die Schilumim, die Juden und die Zionisten bewußt als Zielscheibe für Angriffe gedient haben, um von inneren Problemen in der DDR abzulenken. Die ostdeutsche Regierung erkannte nun auch das Potential des Nahostkonflikts für die Duchsetzung der eigenen Interessen. Neben außenpolitischen Vorteilen erhoffte sich das DDR-Regime von den Außenbeziehungen, vor allem mit Entwicklungsländern, auch eine Verbesserung seiner Stellung und seines Ansehens in der eigenen Bevölkerung. ${ }^{25}$

22 DawAR (Tel Aviv) vom 30. 8. 1953; MA'ARIv (Tel Aviv) vom 27. 8. 1953.

23 Dawar (Tel Aviv) vom 24. 11. 1953.

24 Yachil an den Premierminister, den Außenminister und den Generaldirektor vom 22. 7. 1953; Sharett an Yachil vom 30. 7. 1953, ISA, 2413/13.

25 JACOBSEN, Strategie- und Schwerpunkt, S. 302-305; LAMM/KUPPER, DDR und Dritte Welt, S. 50-52; AUSSENPOLITIK DER DDR; BÜTTNER/HÜNSLER, Die politischen Beziehungen; END, Zweimal deutsche Außenpolitik; KUPPER, Die Tätigkeit der DDR; SCHLECKER/ HACKER, Einmischungen; STEPPAT, Die arabischen Staaten, S. 619-654; KRISENHERD NaH-Ost; Trmm, The Middle East Policy, S. 160-175; SchwanITZ, „Israel ja, Zionismus nein“; Die OSTPOLITIK DER BRD. 
In den folgenden Jahren sollte die DDR als Wegbereiterin der "deutsch-arabischen Freundschaft" und als scharfe Gegnerin Israels auftreten. Mit arabischer Hilfe versuchte sie, die internationale Isolierung zu durchbrechen. Die Außenpolitik der DDR war aber auch von innenpolitischen Bedürfnissen bestimmt. Pankows kurz- und langfristige Politik beruhte ganz auf nüchterner Beurteilung der eigenen Interessen. ${ }^{26}$ Die antijüdischen Kampagnen in der Sowjetunion, der Tschechoslowakei und anderen kommunistischen Ländern fanden auch eine Entsprechung in der DDR: Einzelne jüdische Bürger wurden verhaftet und schikaniert. Andere setzten sich rechtzeitig in den Westen ab, darunter der bereits erwähnte Leo Zucker, das Volkskammermitglied Julius Meyer sowie die Vorsitzenden der jüdischen Gemeinden in Leipzig und Erfurt. ${ }^{27}$

Die Verfolgungen hinterließen offensichtlich einen starken Eindruck auf Ben Gurion, wie ein entsprechender Tagebucheintrag zeigt. ${ }^{28}$ Das prominenteste Opfer der Verhaftungswelle in der DDR war das Zentralkomiteemitglied Paul Merker, der zwar nicht jüdischer Abstammung war, jedoch die Entschädigung von Juden unterstützte. Ein Prozeß gegen ihn fand aber nicht statt, und 1956 wurde er freigelassen. Hans Mayer spekuliert, daß gesellschaftspolitische Gründe den Prozeß verhinderten: Nach Hitler konnten sich die deutschen Kommunisten keinen antijüdischen Schauprozeß leisten. Ein anderer Grund war, Mayer zufolge, die große Zahl der für das DDR-Regime unersetzlichen jüdischen Kollaborateure. ${ }^{29}$ Aufschlußreich ist auch folgende Information von Livneh: Einen Tag vor seiner Flucht soll Mayer einen Brief von Mordekhay Oren, einem Aktivisten der linken israelischen Mapam-Partei erhalten haben. Oren wurde später in der Tschechoslowakei verhaftet und diente der Anklage im Slánský-Prozeß als Zeuge. Laut Mayer hatte sich Oren zu Gesprächen mit DDR-Regierungsvertretern über Schilumim in Osteuropa aufgehalten. ${ }^{30}$ Auch Ostdeutschland scheint die Schilumimoption erwogen zu haben, verwarf sie aber schließlich. Die antiisraelische Linie versprach mehr Vorteile. In der DDR wie in anderen osteuropäischen Ländern sagte man "Zionisten" und meinte die "Juden".

Zur komplexen Realität der DDR gehörte neben der strafrechtlichen Verfolgung ehemaliger Nationalsozialisten und der zeitweiligen Verurteilung des Antijudaismus als Dauerthema der SED-Propaganda auch die Wiedereingliederung ehemaliger NS-Offiziere und hoher Beamter mit nationalsozialistischer Vergangenheit in die Volksarmee und in verschiedene Staatsämter. Linke Vertreter aus Israel und jüdische Linke wurden in Ostberlin mit offenen Armen empfangen, Beziehungen zu potentiellen jüdischen Sympathisanten gefördert. Die Regierung der DDR bekundete zudem Interesse am Handel mit Israel, berichtete über die

${ }^{26}$ Erster Sekretär Speiser an das Außenministerium in Berlin betr. Material über Israel vom 14. 11. 1955, PA, Außenministerium der DDR Berlin, A17063. Diese Akte enthält einen Großteil der ostdeutschen Argumente gegen Israel.

27 Livneh an die Abteilung Osteuropa vom 15.1. 1953, ISA, 2538/21b; Livneh an das Außenministerium vom 21. 1. 1953, ISA, 2413/1; Livneh an die Abteilung Osteuropa vom 3. 2. 1953, ISA, 2387/22; Livneh an die Konsularabteilung vom 13. 3. 1953, CZA, L47/45.

28 Tagebucheintrag vom 16. 3. 1953, BGD, BGA.

29 Hans Mayer an den Autor vom 28. 1. 1989.

30 Livneh an die Abteilung Osteuropa vom 25. 3. 1953, ISA, 2511/17a. 
angebliche Teilnahme Israels an der Leipziger Messe und kaufte israelische Produkte. Etwas Mysteriöses haftet dem Fleischimport durch die sich in israelischem Besitz befindliche eritreische Firma INCODA an. Die Firma schickte sogar einen Vertreter in die DDR, der über lebhaftes Interesse an Handelsbeziehungen mit Israel berichtete. ${ }^{31}$ Livneh wies auf den Umstand hin, daß Pankow die Beziehungen zu dem von ihm geführten Konsulat nicht abgebrochen habe und daß die jüdischen Gemeinden der DDR weiterhin Kontakte mit dem westdeutschen Zentralrat der Juden in Deutschland (ZJD) und dem Jüdischen Weltkongreß (WJC) pflegten.

Solange die Wiedervereinigung Deutschlands einen herausgehobenen Rang auf der internationalen Tagesordnung einnahm, etwa auf den Konferenzen von Berlin und Genf, gingen auch die israelischen Anstrengungen gegenüber Ostdeutschland weiter. Wie wir gesehen haben, versuchte der jüdische Staat auf diesen Konferenzen seine Interessen möglichst gut wahrzunehmen und vor allem auf die Kontinuität der Schilumim im Falle der Wiedervereinigung hinzuwirken. Pankows heftige Kritik an den Schilumim wurde in Israel mit Besorgnis aufgenommen. Das israelische Außenministerium beschloß, die Gespräche mit der Sowjetunion und der DDR wieder zu beleben, die „Friedenskampagne“ der Sowjetunion für den indirekten Zugang zur DDR zu nutzen und Appelts gute Dienste in Anspruch zu nehmen. Am 26. Juni 1955 kam es im Ministerium zu einer Beratung über die Verteidigung des Luxemburger Abkommens und Schilumimforderungen gegenüber der DDR sowie über sowjetische Angelegenheiten. Die Teilnehmer, darunter auch der Außenminister, einigten sich darauf, die Aktivitäten gegenüber der DDR wiederaufzunehmen und gleichzeitig einen Rückzug des Westens von proisraelischen Standpunkten zu verhindern. ${ }^{32}$

Doch es sollte nicht so kommen, wie es sich die israelische Regierung vorgestellt hatte. Appelt starb, und Adenauers Besuch in Moskau im Herbst 1955 schuf eine neue Konstellation. Dem neuen ostdeutschen Botschafter in Moskau fehlten zudem die persönlichen projüdischen Neigungen seines Vorgängers. Bemerkenswert ist die Besorgnis der israelischen Regierung im Hinblick auf Adenauer. Shinnar befürchtete eine negative Reaktion des Bundeskanzlers, sollte er von den israelischen Avancen gegenüber der DDR erfahren. Zu jener Zeit bot die Politik der DDR-Regierung Israel jedenfalls noch keine Gelegenheit, Bonn zur Aufnahme diplomatischer Beziehungen zu drängen. Genaugenommen wurde der Alleinvertretungsanspruch der Bundesrepublik von Israel nie ausdrücklich anerkannt. Das Thema wurde schlicht gemieden. Die israelische Regierung verlangte von der DDR-Führung eine öffentliche Erklärung im Sinne von Adenauers Erklärung

31 Simcha Eilath an Ilsar vom 11.6. 1956, ISA, 613/11. Eilath war möglicherweise ein Offizier des israelischen Geheimdienstes. Der ehemalige Direktor von INCODA, Asher BenNathan, bestritt kategorisch irgendwelche Handelsbeziehungen zwischen der Firma und der DDR in der Vergangenheit: Interview vom August 1989.

32 SHARETT, Yomanim, Bd. 5, Eintrag vom 26. 6. 1955; Der Leiter der Abteilung Westeuropa an den Minister und den Generaldirektor vom 27.6. 1955, ISA, 2511/17b; der Generaldirektor an Shinnar vom, 28.6. 1955, ISA, 613/11; Abteilung Osteuropa an den israelischen Botschafter in Moskau vom 29. 6. 1955 mit Text der Note, die an die Vertreter der Sowjetunion auszuhändigen war, ISA, 2511/17b. 
vom September 1951, und solange sie ausblieb, verzichtete sie auf offizielle Kontakte mit Ostdeutschland. Auf inoffizieller Ebene erklärte sich die israelische Regierung hingegen mehrmals zu Gesprächen mit DDR-Vertretern bereit und unternahm diesbezüglich auch konkrete Vorstöße. Die Frage war nun, wie und bei welcher Gelegenheit dies der Bundesregierung mitgeteilt werden sollte.

Doch vorläufig gaben weder die Sowjetunion noch die DDR konkrete Veranlassung für ein solches "Geständnis". Der israelische Botschafter in der Sowjetunion, Yaakov Avidar, überreichte dem als Hardliner gefürchteten sowjetischen Vizeaußenminister Valerian Zorin eine offizielle Note in Sachen DDR. Dieser verwies ihn an den Botschafter der DDR mit der Begründung, er könne nicht für die DDR sprechen. Auf den Einwand, frühere Noten an jene Adresse seien unbeantwortet geblieben, meinte Zorin ironisch, keine Antwort sei nicht unbedingt eine negative Antwort. ${ }^{33}$ Ein Vermittlungsgesuch an den sowjetischen Botschafter in Israel schlug ebenfalls fehl. Am 3. August 1955 traf Avidar den DDR-Geschäftsträger in Moskau, Christoph Seitz. Der provisorische, niederrangige Stellvertreter des Botschafters war über die Israelpolitik seiner Regierung vermutlich nicht besonders gut informiert: Er erklärte, sein Land sei an freundschaftlichen Beziehungen zu Israel interessiert und falls die Schilumim ein Hindernis darstellten, sei diese Angelegenheit zu klären. In Wirklichkeit dürfte es die DDR-Führung vorgezogen haben, mit dem Staat Israel keine Beziehungen zu unterhalten. Ein gutes Verhältnis mit arabischen Staaten versprach eindeutig mehr Vorteile. Am 2. Dezember 1955 wurde der neue Botschafter der DDR in Moskau, Johannes König, angewiesen, dem israelischen Vertreter eine Note zu überreichen, nachdem er die Vertreter der arabischen Staaten über deren Inhalt informiert hatte. ${ }^{34}$

Ein paar Monate später bat ein israelischer Verein beim Außenministerium der DDR um Photographien und biographische Daten von führenden Politikern der DDR. Die Bitte wurde „in Anbetracht unserer Beziehungen zu den arabischen Ländern und der zugespitzten Lage im Nahen Osten" abgewiesen. ${ }^{35}$ Ein Zeitungsartikel über die angebliche ostdeutsche Anerkennung des Staates Israel wurde vom Regierungssprecher in Ostberlin unverzüglich dementiert. ${ }^{36}$ Das Außenministerium der DDR war offensichtlich bemüht, die arabischen Staaten nicht zu verärgern und sie ständig auf dem laufenden zu halten. Als ein DDR-Vertreter gegenüber einem israelischen Journalisten erklärte, es liege bislang noch kein offizielles israelisches Gesuch an die Regierung der DDR in Sachen Schilumim vor, erwog die israelische Regierung dies nachzuholen. ${ }^{37}$ Doch eine Note der DDR setzte diesen Erwägungen ein jähes Ende. Im undatierten und unbetitelten Dokument wurde erklärt, daß die DDR die Überreste des Faschismus im eigenen Land restlos beseitigt habe und auch gegen den Faschismus im Ausland ankämpfe,

33 Avidar an die Abteilung Osteuropa vom 7. 7. 1955, ISA, 2511/17b.

34 Das Außenministerium der DDR in Berlin an Botschafter Johannes König in Moskau vom 2. 12. 1955, PA, Außenministerium der DDR, Nr. A00682.

35 Hauptabteilung V/2 an das Büro des Staatssekretärs vom 12. 6. 1956, PA, Außenministerium der DDR, A 17594 Berlin.

36 MA'ARIV (Tel Aviv) vom 19.6. 1956. Die Akten des DDR-Außenministeriums enthalten nur dürftige Informationen über die Politik gegenüber Israel.

37 Ilsar an Naor vom 15. 12. 1955, ISA, 613/11. 
was im Interesse des jüdischen Volkes liegen müsse. Die DDR kümmere sich überdies um die Interessen der jüdischen Gemeinden auf ihrem Territorium und habe berechtigte Personen entschädigt. ${ }^{38}$ Obwohl die Note kein einziges Wort über Schilumim enthielt, sorgte sie in israelischen Regierungskreisen für große Aufregung. Manche Regierungsvertreter, darunter auch Golda Meir, interpretierten sie als ostdeutsche Anerkennung Israels als Vertreter des jüdischen Volkes. ${ }^{39}$ Das Außenministerium war sich unschlüssig über die Behandlung der Note und ob Adenauer zu unterrichten sei. Shinnar war dagegen und begründete dies mit der Spannung zwischen beiden deutschen Staaten und den möglichen schädlichen Auswirkungen auf die deutsche öffentliche Meinung.

Von der DDR-Note ermutigt, ließ die israelische Regierung am 20. April 1956 eine weitere Note folgen, in der sie die Rückerstattung der Wiedereingliederungskosten von Überlebenden des Holocaust und Entschädigung für ehemalige Bewohner des derzeitigen Gebietes der DDR verlangte, die nun über die ganze Welt verstreut seien. Botschafter König wies die Forderungen kategorisch zurück mit der Begründung, die DDR sei nicht verantwortlich für die NS-Verbrechen, da sie sich nicht als Nachfolgerin des Dritten Reiches betrachte. Die eigentliche Erbin sei die Bundesrepublik, wo ehemalige Nationalsozialisten lebten und regierten. ${ }^{40}$ Die schriftliche Antwort erfolgte erst rund drei Monate später, am 9. Juli, in Form einer undatierten Note, in der festgehalten wurde, daß keine Veranlassung bestehe, die in der Note vom 28. Dezember 1955 zum Ausdruck gebrachten Standpunkte zu ändern. ${ }^{41}$ Die Israelis waren ungehalten. Offenbar hatte man aus der früheren Note mehr herausgelesen, als wirklich dringestanden hatte, nämlich das was die DDR seit 1952 als Vorwand gegen Schilumim stets vorgebracht hatte. Eine weitere israelische Note, in der die israelische Regierung ihrer Enttäuschung Ausdruck gab und sich das Recht vorbehielt, in Zukunft auf ihre Forderungen zurückzukommen, wurde zwar verfaßt, aber möglicherweise nicht übergeben. ${ }^{42}$

Die Suezkrise war für die DDR eine unverhoffte Gelegenheit für antiwestliche Propaganda bei gleichzeitiger Beweihräucherung der arabischen Seite. Erste Propagandasalven trafen England und Frankreich, doch allmählich geriet auch Israel unter Beschuß. ${ }^{43}$ Die Attacken nahmen an Schärfe zu und bezogen auch jüdische Sprecher mit ein, um ihnen den Charakter der „Kritik aus eigenen Reihen " zu verleihen. ${ }^{44}$ Ihren Höhepunkt erreichten die DDR-Propagandaoffensiven anläßlich des Adenauer-Ben Gurion-Gipfels und während des Eichmannprozesses. Das

38 Text der Note vom 28. 12. 1955, ISA, 2511/17b.

39 Scheck an den Generaldirektor vom 22. 1. 1956; Ilsar an Shinnar vom 25. 1. 1956, ISA, $613 / 11$.

40 Letzte Fassung der Note verm. vom 14.3. 1956, ISA, 613/11; Avidar an die Abteilung Osteuropa vom 20. 4. 1956, ISA, 2511/17b; Ilsar an Shinnar vom 24. 4. 1956, ISA, 613/11.

41 ISA, 613/11.

42 Entwurf der Note o.D., ISA, 613/11.

43 TIMM, Israel in den Medien, S. 160-161; MERTENS, Staatlich propagierter Antisemitismus, S. 142.

44 Arnold Zweig an Werner Pinkus, Deutschlandsender vom 14. 6. 1959, ISA, 3099/21; Ostdeutschlandbericht von John Peet für E. Berent vom 26.11. 1959, LBI, Council of Jews from Germany, General Correspondence, 1960 - December 1961, M/2/1A. 
westdeutsch-israelische Rüstungsgeschäft von 1964/65 bot der DDR sodann eine einmalige Gelegenheit für propagandistischen Profit: Den „westdeutschen Imperialisten " und dem Staat Israel konnte vorgeworfen werden, den vitalen Interessen des „arabischen Volkes“ zu schaden. ${ }^{45}$ Bonn rüste Israel auf, das die arabischen Bestrebungen für Selbstbestimmung unterdrücke und die nationalen Interessen der Araber mißachte, meinten ostdeutsche Sprecher und fügten hinzu, daß Israel den Interessen westdeutscher Kapitalisten diene, die neokapitalistische Ausbeutung nahöstlicher Völker betreibe, und die Bundesrepublik den jüdischen Staat mit Waffen für seine expansionistische Politik beliefere. Ostberlin stellte Bonn und Jerusalem als gemeinsamen Feind der Araber und sich selbst als deren besten Freund und Verbündeten dar: die DDR unterhalte zu keinem dieser Feinde Beziehungen, während sie sich um den Dialog mit den Staaten im Nahen Osten bemühe. Ein weiterer ostdeutscher Vorwurf gegen Westdeutschland betraf die Ruinierung der „traditionellen deutsch-arabischen Freundschaft“. Vor allem die eigenen Interessen vor Augen, präsentierte sich die DDR als Vorkämpferin für die Sache der Dritten Welt und gegen „reaktionäre Kräfte“. Der Skandal rund um das Rüstungsgeschäft $\mathrm{zwischen} \mathrm{der} \mathrm{Bundesrepublik} \mathrm{und} \mathrm{Israel} \mathrm{verschaffte} \mathrm{dem} \mathrm{Staats-}$ ratsvorsitzenden Ulbricht 1965 schließlich eine Einladung nach Kairo.

Walter Ulbrichts Besuch in der Vereinigten Arabischen Republik (VAR) fand zwischen dem 24. Januar und dem 2. Februar 1965 auf Einladung von Präsident Nasser statt. Die Staatsvisite des DDR-Staatsratsvorsitzenden in Ägypten sorgte für Aufruhr in beiden deutschen Staaten. Als Geste gegenüber den ägyptischen Gastgebern übten die ostdeutschen Gäste und Ulbricht persönlich Kritik an Israel und stellten sogar das Existenzrecht des jüdischen Staates in Frage. ${ }^{46}$ Darauf kritisierte die kommunistische israelische Tageszeitung Kol Ha'am am 4. März die SED-Führung und deren Äußerungen in Ägypten mit dem Hinweis, daß Israel weder von Imperialisten gegründet worden sei noch ihnen als Brückenkopf gegen die arabischen Staaten diene. ${ }^{47}$ Ulbrichts Worte trugen zum Zerfall der kommunistischen Partei Israels bei. Während die Sowjetunion bei all ihren Attacken gegen Israel stets Wert darauf legte, das Existenzrecht des jüdischen Staates zu betonen, verfolgte die DDR den eigenen Interessen zuliebe offensichtlich eine radikalere Politik.

\section{Antiisraelismus}

Die israelische Politik gegenüber der DDR machte einen verworrenen Eindruck. Ben Gurion, Golda Meir und Levi Eschkol waren nicht bereit, die Weigerung der DDR, sich zur Mitverantwortung für die NS-Verbrechen zu bekennen, hinzunehmen und sich mit der Ablehnung Ostdeutschlands von Schilumim und Entschädigungszahlungen abzufinden. ${ }^{48}$ Die israelische Diplomatie hegte der DDR

45 TIMM, Hammer, S. 141-146.

46 DiTTMAR, DDR und Israel, S. 848-849; TIMM, Israel in den Medien, S. 163-164.

47 Notiz vom 4. 3. 1965, ISA, 3531/25.

48 Ben Gurion an Golda Meir und Levi vom 3. 11. 1960, BGA, Correspondence File 1960; 
gegenüber gemischte Gefühle. Ihr Verhältnis zum ostdeutschen Staat war zwar nicht offen feindselig, aber doch von Kühle und Groll geprägt. Dabei neigten die israelischen Diplomaten in Osteuropa, wo die Kontakte und Gespräche zwischen beiden Seiten stattfanden, wahrscheinlich zu mehr Flexibilität. Es kam vor, daß der ostdeutsche Ansprechpartner jüdischer Abstammung war und sogar Verwandte in Israel hatte. Einige DDR-Vertreter hegten zudem persönliche Sympathien für den jüdischen Staat. Die ostdeutsche Führung war jedenfalls gegen direkte Kontakte mit israelischen Vertretern, und falls sie sich nicht vermeiden ließen, hatten die ostdeutschen Diplomaten Anweisung, über die Bundesrepublik zu sprechen und zu betonen, daß die DDR an Kontakten mit dem Staat Israel nicht interessiert sei. ${ }^{49}$ Wenn sich die Möglichkeit ergab, von Israel zu profitieren oder wenn den eigenen Interessen Schaden drohte, zögerten ihre Diplomaten hingegen nicht, mit den Israelis Kontakt aufzunehmen.

Die Israelis ließen jeweils kleine Versuchsballone aufsteigen. 1961 machten vier der fünf israelischen Gesandtschaften in Osteuropa Vorschläge zu kleinen Annäherungsgesten. Livneh steuerte aus Prag eine längere Abhandlung bei, in der er unter anderem die Bundesrepublik kritisierte und zu einem Flirt mit der DDR riet. ${ }^{50}$ Wie ist es zu erklären, daß vier von fünf Gesandtschaften im selben Jahr zu ähnlichen Schlußfolgerungen gelangten, davon drei im Oktober 1961? Waren es oppositionelle Regungen gegenüber der doktrinären und unflexiblen Politik der Hardlinerin Meir oder schlicht eine verbreitete Einsicht? Ein Beispiel für die harte Linie war das hartnäckige Festhalten am Besuchsverbot israelischer Dozenten in der DDR. Andererseits konnten die israelischen Behörden die Besuche linker Vertreter in Ostberlin nicht verhindern, schon gar nicht, wenn es sich um Knessetmitglieder handelte.

Die israelische Regierung hatte zwar beschlossen, auf den Handel mit der DDR zu verzichten. ${ }^{51}$ In Wirklichkeit ging er aber trotzdem weiter. Israel verkaufte der DDR Zitrusfrüchte, Chemikalien und Dünger. Was der jüdische Staat in der DDR kaufte, ist unbekannt. Die alltäglichen Bedürfnisse hatten Vorrang vor der Umsetzung doktrinärer Entscheidungen. Zwar beugte sich der ostdeutsche Handel dem arabischen Wirtschaftsboykott, doch das Handelsvolumen zwischen der DDR und Israel war ohnehin gering. Ostdeutschland hätte den Handel mit Israel sicher gerne erweitert, wenn es die politischen Bedingungen erlaubt hätten. Jüdische Organisationen versuchten ihr Glück mit Gesprächsinitiativen gegenüber der DDR aus der Erkenntnis heraus, daß der westliche Boykott in eine Sackgasse führte. Dies blieb jedoch ohne Erfolg.

Die Gründung der DDR hatte bei einigen Israelis, auch bei offiziellen Vertretern, gewisse Hoffnungen geweckt. Doch schon nach kurzer Zeit gesellte sich die

der Leiter der Abteilung Osteuropa an den Chargé d'Affaires in Prag vom 20.12. 1961, ISA, 3309/3.

49 Abteilungsleiter Simons an den Gesandten Georg Stibi in Prag vom 2. 2. 1960, PA, DDRAußenministerium, A 11926 Berlin.

50 Livneh an die Abteilung Osteuropa vom 31. 10. 1961, ISA, 3309/3.

51 Das Datum des Regierungsbeschlusses ist unklar: E.P. Cohen an den Regierungssekretär Katriel Katz vom 17.11. 1959, ISA, 5519/3. 
Republik zu den schlimmsten der nicht-arabischen und nicht-moslemischen Feinde des jüdischen Staates. Die DDR-Führung hatte es in der Tat nicht leicht: Der von den unmittelbaren Nachbarn beargwöhnte und nicht geschätzte ostdeutsche Staat war dazu gezwungen, die Bewegungsfreiheit der eigenen Bevölkerung einzuschränken und gleichzeitig Normalität vorzugaukeln, was nahezu unmöglich war. Die Außenbeziehungen dienten ihr dabei als Instrument für innenpolitische Kampagnen. Im DDR-Pantheon des Bösen, der Feinde des globalen Fortschritts und des sozialistischen Deutschland figurierte der Staat Israel zusammen mit der Bundesrepublik und den USA an vorderster Stelle.

Die Feindseligkeit gegenüber Israel sowie die zahllosen Beschimpfungen und glatten Lügen, mit denen Israel in den DDR-Dokumenten überhäuft wird, sind erschreckend. Daß das jüdische Volk als solches unerwähnt bleibt, liegt möglicherweise schlicht daran, daß die Begriffe „Juden“ und „Judentum" durch „Zionisten" und "Zionismus" ersetzt wurden. So gelang es einigen ostdeutschen Politikern, zwei Fliegen mit einer Klappe zu schlagen. Bewußt oder unbewußt, nutzten sie den zwar latenten, aber durchaus vorhandenen Antijudaismus in der Bevölkerung zur Förderung staatlicher Interessen und zur Ablenkung vom umstrittenen Regime. Einige Dokumente, deren Inhalt an Vorurteile und unbegründete Verunglimpfungen grenzt, erwecken den Eindruck, als hätten die Autoren geradezu auf eine Gelegenheit gewartet, die Juden anzugreifen. Wie auch immer, die doktrinäre und starre Linie der DDR offenbart gewisse pathologische Züge. Die Weigerung, auf Schilumim- und Entschädigungsforderungen einzugehen, zahlte sich sowohl wirtschaftlich als auch politisch aus. In wirtschaftlicher Hinsicht, konnte das Regime das jüdische Eigentum für eigene Zwecke behalten und Ausgaben sparen. Die NS-Beute wurde kurzerhand verstaatlicht. Politisch ergab sich dadurch die Gelegenheit, aus der auch durch die Hallsteindoktrin bewirkten Isolierung auszubrechen: Durch die Verurteilung der westdeutschen Zahlungen an die Juden schloß sich die DDR der entsprechenden arabischen Kritik an und präsentierte sich als Staat, der sich weigert, einen Feind der Araber wirtschaftlich zu unterstützen. ${ }^{52}$ Anfänglich unbedeutend, verwandelte sich die Weigerung der DDR, für Schilumim und andere Entschädigungsforderungen einzutreten, allmählich zu einem Hauptfaktor der ostdeutschen Außenbeziehungen.

Auch die israelische Haltung gegenüber der DDR war starken Veränderungen unterworfen. Unter dem Eindruck des Kalten Krieges und der ideologischen Spaltung der Welt rückte Israel vollständig ins westliche Lager, bei anhaltender Deutschfeindlichkeit der israelischen Öffentlichkeit. Vorurteile abbauende Faktoren, wie Schilumim und Entschädigung sowie die sich entwickelnden vielfältigen beiderseitigen Beziehungen mit der Bundesrepublik, fehlten im Verhältnis zwischen Israel und der DDR. Daran konnte auch der vorgeschobene Antifaschismus Ostberlins nichts ändern. Der fast zwanghafte, weit über die politischen Erfordernisse hinausgehende Antiisraelismus der DDR war den Israelis nicht entgangen. Ihn nur dem Antisemitismus anzulasten, wäre jedoch zu einfach. Die Wurzeln des ostdeutschen Antisemitismus lagen tiefer und waren nur ein kleiner Bestandteil

52 GosCHLER, Paternalismus und Verweigerung, S. 93-117. 
des Antiisraelismus. Entsprechend lag auch der ostdeutsche Antiisraelismus nur zum Teil im Antizionismus begründet.

Außenpolitische Erfordernisse, Klaustrophobie, enttäuschte Erwartungen von freundschaftlichen Beziehungen zu Israel, der Einfluß des Stalinismus und andere Faktoren ergaben eine politische Mischung, die den Eindruck vermittelt, daß der „erste deutsche sozialistische Staat" den Antiisraelismus zur Staatsräson erhoben habe. 